\title{
Mitomicina C colírio como tratamento para granuloma piogênico em cavidade anoftámica
}

\author{
Mitomicyn Ceye drops as alternative treatment for pyogenic granuloma \\ in anophthalmic socket
}

\author{
Hérika Danielle de Miranda Santos ${ }^{1}$ \\ Thatiana Almeida Pereira Fernandes ${ }^{2}$ \\ Cristiano Menezes Diniz ${ }^{3}$ \\ Wellington Tadeu Monteneg'ro ${ }^{4}$ \\ Ana Rosa Pimentel Figueiredo ${ }^{5}$
}

Trabalho realizado no Serviço de Plástica Ocular do Hospital São Geraldo - Universidade Federal de Minas Gerais - UFMG - Belo Horizonte (MG) - Brasil.

Fellow do Serviço de Plástica Ocular do Hospital São Geraldo - Universidade Federal de Minas Gerais UFMG - Belo Horizonte (MG) - Brasil.

Fellow do Serviço de Plástica Ocular do Hospital São Geraldo - UFMG - Belo Horizonte (MG) - Brasil.

Médico do Serviço de Plástica Ocular do Hospital São Geraldo - UFMG - Belo Horizonte (MG) - Brasil.

Doutor em Oftalmologia; Médico do Serviço de Córnea Catarata e Doenças Externas Oculares do Hospital São Geraldo - UFMG - Belo Horizonte (MG) - Brasil.

Professora do Departamento de Oftalmologia da Faculdade de Medicina - UFMG. Chefe do Serviço de Plástica Ocular do Hospital São Geraldo - UFMG - Belo Horizonte (MG) - Brasil.

Endereço para correspondência: Hérika Danielle de Miranda Santos. Av. Assis Chateaubriand, 233/105 Belo Horizonte (MG) CEP 30150-100

Recebido para publicação em 11.04.2005

Última versão recebida em 06.02.2006

Aprovação em 02.05.2006

Nota Editorial: Depois de concluída a análise do artigo sob sigilo editorial e com a anuência do Dra. Ana Estela Besteti Pires Ponce Sant'Anna sobre a divulgação de seu nome como revisora, agradecemos sua participação neste processo.

\section{RESUMO}

Objetivo: Avaliar a eficácia do uso tópico de colírio de mitomicina $\mathrm{C}$ $0,02 \%$ (MMC 0,02\%) no tratamento de pacientes com granuloma piogênico em cavidade anoftálmica. Métodos: Seis pacientes portadores de granuloma piogênico em cavidade anoftálmica foram submetidos ao tratamento com mitomicina $\mathrm{C} 0,02 \%$ instilada quatro vezes ao dia durante ciclos quinzenais e intervalo de 15 dias entre os ciclos. Os pacientes foram acompanhados semanalmente. Resultados: Foram acompanhados seis pacientes dos quais um apresentou resolução do granuloma piogênico ao final do primeiro ciclo de uso da MMC 0,02\%. Em dois pacientes a MMC 0,02\% foi eficaz na resolução da lesão após a segunda semana de tratamento (primeiro ciclo). Dois pacientes apresentaram resolução completa do granuloma piogênico na quinta semana de uso da MMC 0,02\% (início do segundo ciclo). Este fármaco foi ineficaz em apenas um paciente que persistiu com a lesão após dois ciclos de tratamento. Não foram observadas complicações decorrentes do uso de MMC 0,02\% em nenhum dos pacientes. Conclusão: A MMC 0,02\% tópica mostrou-se eficaz no tratamento de granuloma piogênico em cavidade anoftálmica, entretanto, diante de casuística pequena, torna-se necessário estudo mais amplo, comparando a eficácia da MMC 0,02\% com drogas já utilizadas no tratamento desta lesão, tais como os esteróides e antimetabólicos.

Descritores: Mitomicina/uso terapêutico; Granuloma piogênico/quimioterapia; Cavidade anoftalmia; Inflamação

\section{INTRODUÇ̃̃̃O}

A mitomicina $\mathrm{C}$ (MMC) é um agente alquilante inibidor da síntese de DNA dependente do RNA, da síntese protéica em células de crescimento rápido e da proliferação fibroblástica ${ }^{(1-2)}$ ao nível episcleral. Seu mecanismo de ação mimetiza a radiação ionizante com uma ação que se prolonga após a interrupção do tratamento ${ }^{(3-4)}$.

A MMC tópica diluída atualmente vem sendo utilizada em oftalmologia no pré-operatório da exérese de pterígios recidivados ${ }^{(5-7)}$, como coadjuvante em casos selecionados de trabeculectomias ${ }^{(8-12)}$, como modulador em cirurgias refrativas $^{(4)}$, no tratamento das neoplasias intra-epiteliais corneanas $^{(13)}$, da melanose conjuntival primária adquirida com atipia ${ }^{(14-15)}$, nas cirurgias de dacriocistorrinostomias ${ }^{(16-17)}$ e na atenuação dos períodos de exacerbação da ceratoconjuntivite primaveril ${ }^{(18)}$. Um estudo recente realizado pelo Serviço de Córnea, Doenças Externas Oculares, Catarata e Cirurgia Refrativa do Hospital São Geraldo - UFMG, confirmou a eficácia da MMC 
0,02\% no tratamento de granulomas piogênicos córneo-conjuntivais $^{(19)}$. Este trabalho, por sua vez, vem testar a eficácia da MMC tópica na concentração de $0,02 \%$ no tratamento de granuloma piogênico em cavidades anoftálmicas, ainda não descrito na literatura.

Contudo, a toxicidade da MMC representa uma das maiores preocupações em relação ao seu uso em oftalmologia e será também abordada neste estudo. Por outro lado, um traba1 ho ${ }^{(20)}$ revela que o uso da MMC subconjuntival, na cabeça do pterígio, um mês antes da sua excisão, oferece bons resultados sem grandes complicações.

Baseando-se na ação inibitória da síntese de fibroblastos, na inibição da multiplicação celular pela MMC e na sua eficácia comprovada em várias áreas da oftalmologia, este estudo tem por objetivo avaliar a resposta ao uso tópico desta droga na concentração de $0,02 \%$ em pacientes com granulomas piogênicos em cavidades anoftálmicas, local onde a conjuntiva apresenta particularidades que serão abordadas posteriormente neste estudo.

\section{MÉTODOS}

Seis pacientes sendo quatro mulheres e dois homens, com idade entre 13 a 85 anos (média de 47,9 anos), atendidos no Serviço de Plástica Ocular, Órbita e Vias Lacrimais do Hospital São Geraldo - UFMG.

Os critérios de inclusão deste estudo envolveram indivíduos portadores de lesões clinicamente compatíveis com granuloma piogênico em cavidade anoftálmica, independente da faixa etária, sexo ou das condições da cavidade. Todos os pacientes foram esclarecidos quanto à natureza e efeitos do colírio de MMC e autorizaram a utilização do mesmo através de um termo de consentimento.

Dentre os seis pacientes, quatro foram submetidos à evisceração conseqüente a endoftalmite e dois à enucleação devido a tumor intra-ocular e trauma penetrante.

Quatro usavam a mesma prótese por um período superior a três anos, dos quais três apresentavam uma estética insatisfatória e um razoável. Dois pacientes não eram usuários de prótese.

Clinicamente, nos quatro pacientes eviscerados não foi observada qualquer alteração de fórnice. Entretanto, os dois pacientes enucleados e não usuários de prótese apresentavam algum grau de retração de fórnices.

Em todos os casos, o granuloma piogênico não era uma lesão recidivante, tendo os fórnices inferior e lateral como as principais localizações.

Todos os pacientes do estudo apresentavam sinais inflamatórios de intensidade variada nas cavidades avaliadas. Dentre os sintomas mais comuns, destacaram-se a sensação de corpo estranho e o lacrimejamento. Apenas um paciente permaneceu assintomático.

Os pacientes foram submetidos ao uso tópico de MMC $0,02 \%$ instilada quatro vezes ao dia, em ciclos de 15 dias cada, com pausa quinzenal entre os mesmos, num total máximo de três ciclos. Todos os frascos dos colírios utilizados na pesquisa foram fabricados numa mesma farmácia de manipulação sob supervisão de um único farmacêutico capacitado.

Durante o uso da MMC 0,02\%, todos os pacientes foram avaliados semanalmente, verificando-se a presença de sintomas, além das dimensões das lesões através de biomicroscopia e fotodocumentação. Após conclusão do tratamento e resolução completa da lesão, os pacientes continuaram acompanhamento com freqüência mensal.

\section{RESULTADOS}

Todos os pacientes apresentaram melhora da sintomatologia e redução das dimensões das lesões constatada à biomicroscopia.

Um paciente evoluiu com resolução completa do granuloma piogênico após dez dias de uso da MMC 0,02\%, antes do término do primeiro ciclo (Figura 1, antes e Figura 2, após o

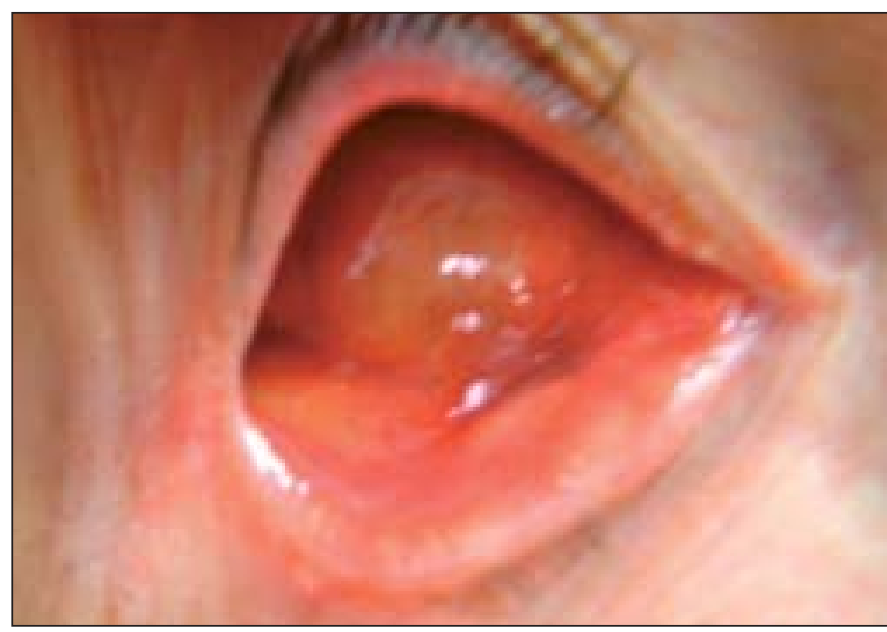

Figura 1 - Paciente 1, antes do tratamento com MMC 0,02\%

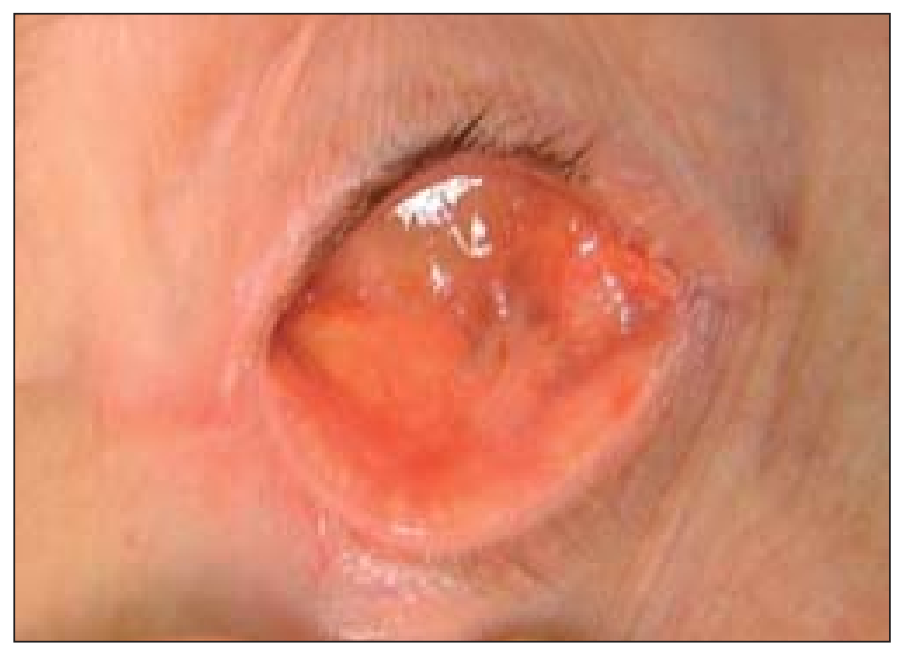

Figura 2 - Paciente 1, após um ciclo de tratamento com MMC 0,02\% 
tratamento). Após a segunda semana de tratamento (primeiro ciclo completo), houve desaparecimento total das lesões em dois pacientes, interrompendo-se o uso da MMC 0,02\%. Na quinta semana de acompanhamento, início do segundo ciclo, observou-se resolução clínica das lesões em dois pacientes (Figura 3, antes e Figura 4, após o tratamento). O segundo ciclo do tratamento foi concluído e o tratamento finalizado nestes pacientes (Tabela 1).

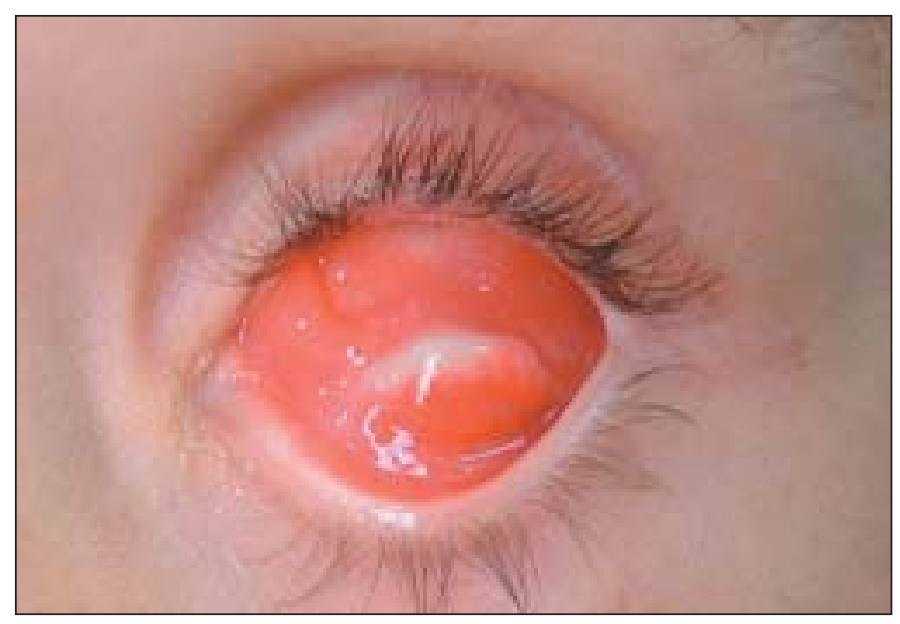

Figura 3 - Paciente 3, antes do tratamento com MMC 0,02\%

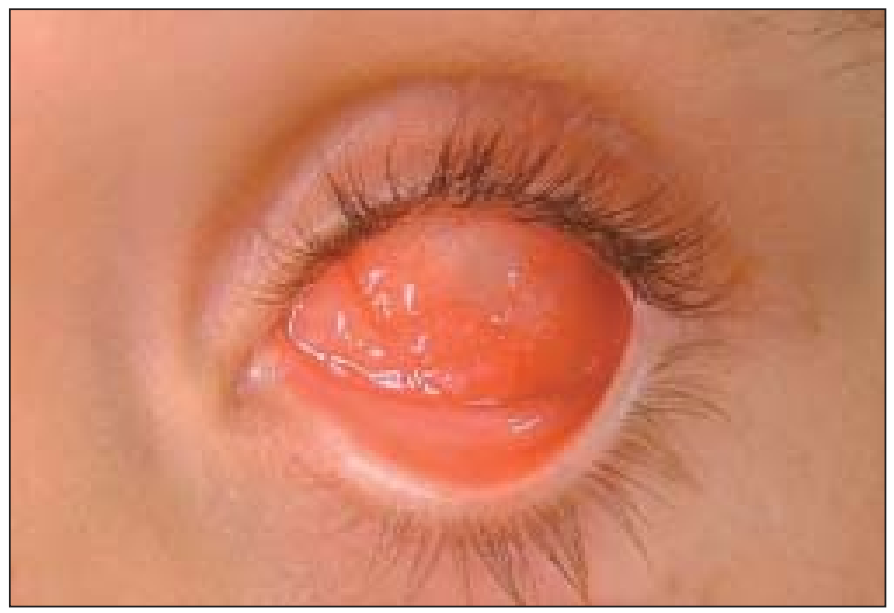

Figura 4 - Paciente 3, após dois ciclos de tratamento com MMC 0,02\%

\begin{tabular}{|lcccc|}
\hline \multicolumn{5}{|c|}{$\begin{array}{c}\text { Tabela 1. Descrição dos pacientes com granuloma piogênico em } \\
\text { cavidade anoftálmica tratados com MMC }\end{array}$} \\
Paciente & .,02\% \\
1 & Sexo & $\begin{array}{c}\text { Uso de } \\
\text { prótese }\end{array}$ & $\begin{array}{c}\text { Ciclos de } \\
\text { MMC }\end{array}$ & $\begin{array}{c}\text { Cura da } \\
\text { lesão }\end{array}$ \\
2 & F & Sim & 1 & Sim \\
3 & F & Sim & 1 & Sim \\
4 & M & Sim & 2 & Sim \\
5 & F & Não & 1 & Sim \\
6 & M & Sim & 2 & Não \\
\hline & F & Não & 2 & Sim \\
\hline
\end{tabular}

Apenas um paciente não respondeu satisfatoriamente ao tratamento com MMC 0,02\% após dois ciclos tendo sido indicada exérese cirúrgica da lesão.

Durante o período de tratamento, não foi observada nenhuma complicação em relação ao uso da MMC 0,02\% em nenhum dos pacientes avaliados neste estudo.

\section{DISCUSS $\tilde{A} O$}

O granuloma piogênico é uma lesão ricamente vascularizada que compromete pele e membranas mucosas ${ }^{(21)}$. É resultante da proliferação reativa de células endoteliais vasculares e tecido de granulação (tecido conectivo proliferativo, fibroblastos e canais vasculares neoformados) ${ }^{(22)}$. No olho, usualmente envolve a conjuntiva, o limbo, as pálpebras e, mais raramente, a córnea ${ }^{(23-24)}$. No presente estudo observou-se a presença desta lesão em cavidades anoftálmicas nos seis pacientes acompanhados.

A perda do globo ocular através da enucleação ou evisceração acarreta deformidades anatômicas e fisiológicas variadas da órbita, comprometendo a relação ideal cavidade-prótese, em consequiência da perda do volume orbitário, da contração dos músculos extra-oculares e da mobilização de tecidos orbitários $^{(25)}$.

Assim como os tecidos já descritos, também as conjuntivas que revestem estas cavidades sofrem modificações decorrentes do constante processo irritativo do complexo mucosa conjuntival-prótese induzindo alterações inflamatórias crônicas e conseqüiente formação de uma fibrose conjuntival progressiva, proliferação de neovasos com a formação dos granulomas piogênicos, acúmulo de secreção e incômodo ao paciente.

As opções de tratamento para o granuloma piogênico incluem esteróides tópicos, crioterapia, reexcisão, transplante de conjuntiva, antimetabólicos tópicos e radiação. O Serviço de Plástica Ocular e Órbita do Hospital São Geraldo - UFMG preconizou o uso da MMC 0,02\% tópica como tratamento de granulomas piogênicos em cavidades anoftálmicas diante da eficácia desta droga já relatada no tratamento de lesões semelhantes no segmento anterior do olho ${ }^{(19)}$.

A MMC é um fármaco não desprovido de efeitos tóxicos sobre o olho, quando utilizado topicamente. Dentre as complicações relacionadas a MMC tópica estão as blefarites, ceratites, edema de córnea, calcificações esclerais, necrose de córnea e esclera, corectopia, catarata madura de início precoce, glaucoma secundário, hipotonia ocular associada à maculopatia clinicamente significativa e até mesmo endoftalmite ${ }^{(6,26)}$. Neste estudo, no entanto, não foram observadas complicações com o uso tópico da MMC 0,02\% (quatro vezes ao dia durante 15 dias num máximo de três ciclos) em nenhum dos pacientes.

Conclui-se que o uso tópico de MMC foi eficaz no tratamento de granulomas piogênicos em cavidades anoftálmicas de seis dos sete pacientes acompanhados. Porém, se faz necessário a realização de trabalhos comparativos e randomiza- 
dos, envolvendo um maior número de casos, a fim de se comparar a eficácia da mitomicina $\mathrm{C}$ tópica com as drogas já usadas tais como os esteróides e os antimetabólicos.

\section{ABSTRACT}

Purpose: To evaluate the efficacy of mitomycin $\mathrm{C}$ eye drops $0.02 \%$ concentration $(0.02 \% \mathrm{MMC})$ in pyogenic granuloma in anophthalmic socket. Methods: Six patients who presented pyogenic granuloma were treated with $0.02 \% \mathrm{MMC}$ four times a day in fifteen-day cycles with intervals of fifteen days between each cycle. The patients were observed every week. Results: Among the six studied patients, one presented a complete resolution of the pyogenic granuloma after a ten-day treatment with $0.02 \% \mathrm{MMC}$ that is, before the end of the first cycle. In two patients, the treatment was successful after two weeks using $0.02 \% \mathrm{MMC}$ (one complete cycle). In other two patients, the resolution of the lesion occurred after five weeks that is, at the beginning of the second cycle. $0.02 \% \mathrm{MMC}$ was not successful in only one patient whose lesion persisted after two cycles of the treatment. None of the patients presented complications after the use of $0.02 \%$ MMC. Conclusion: The topical use of $0.02 \% \mathrm{MMC}$ proved efficacy in the treatment of anophthalmic socket pyogenic granuloma. A small number of patients took part in this study, therefore another randomized and comparative research is necessary in order to compare the efficacy of $0.02 \% \mathrm{MMC}$ with other drugs such as corticosteroids and antimetabolites.

Keywords: Mitomycin/therapeutic use, Granuloma, pyogenic/ drug therapy; Anophthalmos; Inflammation

\section{REFERÊNCIAS}

1. Lee CC, Chen CW, Chao MC. A study of inhibitory effect of mitomycin C on fibroblasts in cell culture. Trans Ophthalmol Soc Republic China. 1986;25: 752-60.

2. Yamamoto T, Varani J, Soong HK, Lichter PR. Effects of 5-fluorouracil and mitomycin $\mathrm{C}$ on cultured rabbit subconjunctival fibroblasts. Ophthalmology. 1990;97(9):1204-10.

3. Crooke ST, Bradner WT. Mitomycin C: a review. Cancer Treat Rev. 1976; 3(3):121-39.

4. Talamo JH, Gollamudi S, Green R, De La Cruz Z, Filatov V, Stark WJ.
Modulation of corneal wound healing after excimer laser keratomileusis using topical mitomycin C and steroids. Arch Ophthalmol. 1991;109(8):1141-6.

5. Rosenthal G, Shoaham A, Lifshitz T, Biedner B, Yassur Y. The use of mitomycin in pterygium surgery. Ann Ophthalmol. 1993;25(11):427-8.

6. Rubinfeld RS, Pfister RR, Stein RM, Foster CS, Martin NF, Stoleru S, et al. Serious complications of topical mitomycin-C after pterygium surgery. Ophthalmology. 1992;99(11):1647-54.

7. Singh G, Wilson MR, Foster CS. Mitomycin eye drops as treatment for pterygium. Ophthalmology. 1988;95(6):813-21.

8. Costa VP, Moster MR, Wilson RP, Schmidt CM, Gandham S, Smith M. Effects of topical mitomycin $\mathrm{C}$ on primary trabeculectomies and combined procedures. Br J Ophthalmol. 1993;77(11):693-7.

9. Chen CW, Huang HT, Sheu MM. Enhancement of IOP control efficacy of trabeculectomy by local application of anticancer drug. In: Acta XXV Concilium Ophthalmologicum Rome. 1986;2:1487-91.

10. Edwards RS. Trabeculectomy combined with cataract extraction: a follow-up study. Br J Ophthalmol. 1980;64(9):720-4.

11. Palmer SS. Mitomycin as adjunct chemotherapy with trabeculectomy. Ophthalmology. 1991;98(3):317-21.

12. Percival SP. Glaucoma triple procedure of extracapsular cataract extraction, posterior chamber lens implantation, and trabeculectomy. $\mathrm{Br} \mathrm{J}$ Ophthalmol. 1985;69(2):99-102.

13. Frucht-Pery J, Rozenman Y. Mitomycin C therapy for corneal intraepithelial neoplasia. Am J Ophthalmol. 1994;117(2):164-8.

14. Demirci H, McCormick SA, Finger PT. Topical mitomycin chemotherapy for conjunctival malignant melanoma and primary acquired melanosis with atypia: clinical experience with histopathologic observations. Arch Ophthalmol. 2000; 118(7):885-91.

15. Frucht-Pery J, Pe'er J. Use of mitomycin $\mathrm{C}$ in the treatment conjunctival primary acquired melanosis with atypia. Arch Ophthalmol. 1996;114(10):1261-4.

16. Hu D, Sires BS, Tong DC, Royack GA, Oda D. Effect of brief exposure to mitomycin $\mathrm{C}$ on cultured human nasal mucosa fibroblasts. Ophthal Plast Reconstr Surg. 2000;16(2):119-25.

17. Yeatts RP, Neves RB. Use of mitomycin C in repeat dacryocystorhinostomy. Ophthal Plast Reconstr Surg. 1999;15(1):19-22.

18. Akpek EK, Hasiripi H, Christen WG, Kalayci D. A randomized trial of low dose, topical mitomycin- $\mathrm{C}$ in the treatment of severe vernal keratoconjunctivitis. Ophthalmology. 2000;107(2):263-9.

19. Montenegro WT, Tanure MAG, Diniz CM, Trindade FC. Mitomicina C colírio como tratamento para granuloma piogênico da superfície corneoconjuntival. Rev Bras Oftalmol. 2002;61(10):721-6.

20. Donnenfeld ED, Perry HD, Fromer S, Doshi S, Solomon R, Biser S. Subconjunctival mitomycin $\mathrm{C}$ as adjunctive therapy before pterygium excision. Ophthalmology. 2003;110(5):1012-6.

21. Ferry AP. Pyogenic granulomas of the eye and ocular adnexa: a study of 100 cases. Trans Am Ophthalmol Soc. 1989;87:327-43; discussion 343-7.

22. Boockvar W, Wessely Z, Ballen P. Recurrent granuloma pyogenicum of limbus. Arch Ophthalmol. 1974;91(1):42-4.

23. De Potter P, Tardio DJ, Shields CL, Shields JA. Pyogenic granuloma of the cornea after penetrating keratoplasty. Cornea. 1992;11(6):589-91.

24. Ferry AP, Zimmerman LE. Granuloma pyogenicum of limbus. Arch Ophthalmol. 1965;74:229-30.

25. Clauser L, Sarti E, Dallera V, Galie M. Integrated reconstructive strategies for treating the anophthalmic orbit. J Craniomaxillofac Surg. 2004;32(5):279-90.

26. Sugar A. Who should receive mitomycin-C after pterygium surgery? Ophthalmology. 1992;99(11):1645-6. 